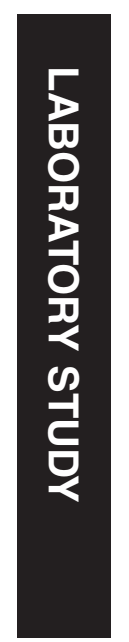

\title{
MicroRNA-125b promotes tumor growth and suppresses apoptosis by targeting DRAM2 in retinoblastoma
}

'Department of Ophthalmology, Xi'an No.4 Hospital, Guangren Hospital of Xi'an Jiaotong University, Xi'an, China

${ }^{2}$ Department of Ophthalmology, First Hospital of Jilin University, Changchun, China

Correspondence: A Li, Department of Ophthalmology, First Hospital of Jilin University, Xinmin Street No. 71, Chaoyang District, Changchun City, Jilin Province 130021, China Tel: +86 4318187 5572; Fax: +86 43188782352 . E-mail: aipengli4@163.com

${ }^{3}$ These authors contributed equally to this work

Received: 21 February 2016 Accepted in revised form: 1 July 2016

Published online:

12 August 2016

\begin{abstract}
Purpose It is known that microRNAs (miRNAs) are a class of small, noncoding RNAs that act as key regulators in various physiological and pathological processes. However, the regulatory mechanisms involving miRNAs in retinoblastoma (RB) remain largely unknown. The miRNA miR-125b is dysregulated in various human cancers such as breast cancer, human hepatocellular carcinoma, ovarian cancer, and colorectal cancer. However, the significance of miR-125b in RB has not been sufficiently investigated. Our objective was to explore the role of the miR-125b in $R B$.

Methods In this study, we measured miR-125b levels using real-time polymerase chain reaction in human $R B$ cell lines, including HXO-Rb44, Y79, SO-RB50, and the normal human retinal pigment epithelial cell line ARPE-19; a total of 38 pairs of primary RB tissues and adjacent noncancerous tissues were also measured. In addition, overexpression of miR-125b in RB cell lines was performed to determine the role of miR-125b in RB.

Results We found that miR-125b is significantly upregulated in RB, and closely associated with tumor cell proliferation and apoptosis. In addition, overexpression of miR-125b apparently promotes $R B$ cell proliferation and migration in vitro. Gain-offunction in vitro experiments further showed that the miR-125b mimic significantly suppressed RB cell apoptosis. A subsequent dual-luciferase reporter assay identified the suppressor gene DRAM2 as direct target of miR-125b.

Conclusions Our data collectively demonstrate that miR-125b is a suppressor
\end{abstract}

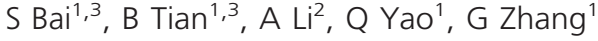
and $\mathrm{F} \mathrm{Li}^{1}$ gene miRNA that can promote $\mathrm{RB}$ cell proliferation and migration by downregulating the suppressor gene DRAM2, indicating that $\mathrm{miR}-125 \mathrm{~b}$ may represent a new potential diagnostic and therapeutic target for RB treatment.

Eye (2016) 30, 1630-1638; doi:10.1038/eye.2016.189; published online 12 August 2016

\section{Introduction}

Retinoblastoma (RB) is the most common intraocular malignant tumor arising in the retina. It mainly occurs in infants, with an incidence of about 1/15 000-1/20 000 live births, translating to $\sim 9000$ new cases every year worldwide. ${ }^{1,2}$ The incidence and mortality rate of retinoblastoma in China have increased rapidly over the past several decades. ${ }^{3}$ Hence, it is imperative to explore the mechanisms by which retinoblastoma is initiated and what factors drive progression. Recently, microRNAs (miRNAs) have been identified as novel molecules that are crucial in cancer development. ${ }^{4,5}$

MiRNAs are small noncoding RNAs that posttranscriptionally modulate gene expression by repressing translation or accelerating mRNA degradation. ${ }^{6,7}$ In general, miRNAs exert their regulatory role on protein-coding gene expression by binding to either full or partial complementary sequences, primarily in the $3^{\prime}$ untranslated region ( $3^{\prime}$ UTR) of target mRNAs. ${ }^{8}$ Numerous studies have shown that miRNAs participate in various biological processes, including cell growth, development, apoptosis, and differentiation. ${ }^{9-12}$ Accumulated studies have revealed that miRNAs may be oncogenic or tumor suppressors, in which oncogenic miRNAs are upregulated, whereas tumor suppressors are downregulated in cancer. ${ }^{13}$ To date, several 
studies have shown that miRNAs are aberrantly downregulated or overexpressed during the retinoblastoma progression, including miR-192, miR-34a, miR-183, and miR-376a. ${ }^{14-17}$ However, the role and function of miRNAs in retinoblastoma are still emerging, and the roles of many other aberrantly expressed miRNAs in retinoblastoma development as well as the mechanisms involved are still unknown.

Previous studies have demonstrated that the expression of miR-125b is deregulated in several types of human cancer. For example, $\mathrm{Xu}$-Bao et al showed that miR-125b is upregulated in prostate cancer and promotes tumor growth through directly targeting eight transcripts, including three key proapoptotic genes, that is, p53, Puma, and Bak1. ${ }^{18}$ Li et al ${ }^{19}$ found that miR-125b levels are significantly higher in non-small-cell lung cancer (NSCLC) cells and target TP53INP1 (tumor protein 53-induced nuclear protein 1), which is involved in the metastasis of NSCLC cells. $\mathrm{Gu}$ et $a \mathrm{l}^{20}$ showed that miR-125b is increased in oral squamous cell carcinoma. Several studies have shown that the expression level of miR-125b is significantly increased in retinoblastoma cells. ${ }^{21,22}$ However, the role and function of miR-125b in retinoblastoma development as well as the mechanisms involved are still unknown.

It is known that miRNAs exert biological functions by regulating specific target genes. To determine the detailed mechanism by which miR-125b promotes retinoblastoma cell growth, DRAM2 (DNA-damageregulated autophagy modulator protein 2), was further identified as one of the putative targets of miR-125b. The DRAM2 gene, also known as TMEM77 (transmembrane protein 77), encodes a 266-amino acid protein containing six putative transmembrane domains. ${ }^{23}$ DRAM2 is localized to lysosomal membranes, ${ }^{24}$ where it initiates the conversion of endogenous LC3-I (microtubule-associated protein light chain 3) to the general autophagosome marker protein, LC3-II (LC3-1/phosphatidylethanolamine conjugate). Previous reports have shown that DRAM2 induces the autophagy process and is an effector molecule critical for p53-mediated apoptosis. ${ }^{24}$ Increasing evidence indicates that DRAM2 may have a part in tumor signaling because it is downregulated in many cancer tissues, such as ovarian cancer. ${ }^{25}$ Previous studies have shown that high expression of DRAM2 could promote apoptosis. However, the expression pattern of the DRAM2 molecule in human retinoblastoma tissue and cells is not clear.

In this study, we demonstrate that miR-125b is upregulated in retinoblastoma tissues, as well as in various retinoblastoma cell lines, including $\mathrm{HXO}-\mathrm{Rb} 44$, Y79, and SO-RB50. Furthermore, we investigated whether
miR-125b may be involved in cell proliferation, migration, and invasion. We looked for unknown target genes of miR-125b and identified DRAM2 was a direct target of miR-125b. In addition, miR-125b functions as a tumor oncogene gene by downregulating DRAM2 expression, providing a potential diagnostic and therapeutic target for the treatment of retinoblastoma.

\section{Materials and methods}

\section{Tissue samples}

A total of 38 pairs of primary retinoblastoma tissues and adjacent noncancerous tissues (NCTs); (located $>3 \mathrm{~cm}$ away from the tumor) were collected from the Xi'an Jiaotong University Affiliated Hospital, Guangren Hospital (Xi'an, China). All subjects were diagnosed and confirmed by a pathologist. None of the subjects received any biotherapy or chemotherapy treatment before recruitment to this study. All of the human material was obtained with informed consent, and the present study was approved by the Ethics Committee of the Guangren Hospital, College of Medicine, Xi'an Jiaotong University.

\section{Cell lines and cell culture}

The human retinoblastoma cell lines, including HXO$\mathrm{Rb} 44, \mathrm{Y} 79$, and SO-RB50, and the normal human retinal pigment epithelial cell line ARPE-19 were purchased from the American Type Culture Collection (ATCC, Manassas, VA, USA). All the cancer cells were cultured in RPMI-1640 medium containing $100 \mathrm{IU} / \mathrm{ml}$ penicillin, $100 \mu \mathrm{g} / \mathrm{ml}$ streptomycin, $20 \mathrm{mM}$ glutamine, and 10\% heat-inactivated fetal bovine serum (FBS). The normal human retinal pigment epithelial cell line ARPE-19 was grown in DMEM medium with $10 \%$ FBS, $10 \mathrm{ng} / \mathrm{ml}$ cholera toxin, $5 \mu \mathrm{g} / \mathrm{ml}$ transferrin, $5 \mu \mathrm{g} / \mathrm{ml}$ insulin, $100 \mathrm{ng} / \mathrm{ml}$ hydrocortisone, and $10 \mathrm{mM}$ 4-(2hydroxyethyl)-1-piperazine ethanesulfonic acid (HEPES). All cells were cultured in a humidified atmosphere of $5 \%$ $\mathrm{CO}_{2}$ at $37^{\circ} \mathrm{C}$.

\section{Cell transfection}

The oligonucleotides of the miR-125b mimic and its negative control (miR-NC), miR-125b inhibitor and its negative control (anti-miR) were purchased from Genepharma Co., Ltd (Shanghai, China). The pcDNA3.1/ DRAM2 plasmid was designed by Genepharma (Shanghai, China). For transient transfection, cells were plated for $16 \mathrm{~h}$ before transfection at $50 \%$ confluence, and then transfected with $25 \mathrm{nM}$ of either the miR-125b mimic or miR-125b inhibitor using Lipofectamine 2000 
(Invitrogen Life Technologies, Carlsbad, CA, USA) according to the manufacturer's instructions. After transfection, the transfection effect was determined by real-time polymerase chain reaction (RT-PCR). For DRAM2 overexpression, $0.5 \mu \mathrm{g}$ of pcDNA3.1/DRAM2 or pcDNA3.1 control vector was transfected into cells using Lipofectamine 2000 (Life Technologies, Carlsbad, CA, USA). Cells were harvested after $48 \mathrm{~h}$ for subsequent experiments.

\section{RNA extraction and RT-PCR}

Total RNA was extracted from cells or frozen tissues using Trizol reagent (Invitrogen Life Technologies). The concentrations of RNA were determined using a NanoDrop ND-1000 instrument (ThermoFisher, Waltham, MA, USA) and aliquots of the samples were stored at $-80^{\circ} \mathrm{C}$. For the reverse transcription (RT) reaction of the miRNA, specific miRNA RT primers were used. U6 small nuclear noncoding RNA served as an internal control. To measure DRAM2 mRNA, cDNA was synthesized using DNA polymerase I and $\mathrm{RNaseH}$, and then purified using QiaQuick PCR extraction kit (Qiagen, Hilden, Germany) according to the manufacturer's instructions. Aliquots of the reaction mixture were used for quantitative PCR with TaqMan $2 \times$ Universal PCR Master Mix (Applied Biosystems, Foster City, CA, USA), using the following conditions: initial denaturation at $95^{\circ} \mathrm{C}$ for $10 \mathrm{~min}$, followed by 40 cycles of $95^{\circ} \mathrm{C}$ for $15 \mathrm{~s}$, $60{ }^{\circ} \mathrm{C}$ for $1 \mathrm{~min}$, and $72{ }^{\circ} \mathrm{C}$ for $45 \mathrm{~s}$. All PCR experiments were performed in triplicate.

\section{Cell proliferation assay}

Cells were seeded in 96-well plates at $6 \times 10^{3}$ cells /well, and the surviving fractions were determined at 1-5 days using the MTT assay, as previously described. ${ }^{26}$ The absorbance was measured with a spectrophotometer (Bio-Rad Laboratories, Hercules, CA, USA) at $570 \mathrm{~nm}$. Each experiment was performed in triplicate. ${ }^{27}$

\section{Cell migration and invasion assays}

$\mathrm{HXO}-\mathrm{Rb} 44$ cells were grown to confluence in 12-well plastic dishes, and treated with the miR-125b mimic and its negative control (miR-NC), or the miR-125b inhibitor and its negative control (anti-miR). Then, $24 \mathrm{~h}$ after transfection, linear scratch wounds (in triplicate) were created on the confluent cell monolayers using a $200 \mu 1$ pipette tip. To remove cells from the cell cycle prior to wounding, cells were maintained in serum-free media. To visualize migrated cells and wound healing, images were taken at different time points. A total of 10 areas were selected randomly from each well, and the cells in three wells from each group were quantified.

For the invasion assays, $24 \mathrm{~h}$ after transfection, $1 \times 10^{5}$ cells in serum-free media were seeded in Transwell migration chambers ( $8 \mu \mathrm{m}$ pore size; Millipore, Corning Inc., Corning, NY, USA). The upper chamber of the Transwell inserts was coated with Matrigel (SigmaAldrich, St Louis, MO, USA). Medium containing 20\% FBS was added to the lower chamber. After $24 \mathrm{~h}$, the noninvading cells were removed with cotton wool. Invasive cells located on the lower surface of the chamber were stained with May-Grunwald-Giemsa stain (SigmaAldrich) and counted using a microscope (Olympus, Tokyo, Japan). Experiments were independently repeated three times.

\section{Apoptosis assay}

An Annexin V apoptosis detection kit (BD Pharmingen, San Diego, CA, USA) was used to assess apoptosis. Following transfection for $72 \mathrm{~h}$, the cells were collected and detected using an Annexin $\mathrm{V}$ fluorescein isothiocyanate kit (FITC) (Biolegend, San Diego, CA, USA) according to the manufacturer's instructions. In brief, cells transfected with the miR-125b mimic or the miR-125b inhibitor were resuspended in $100 \mathrm{ml}$ binding buffer, at a density of $1 \times 106$ cells $/ \mathrm{ml}$, then incubated with Annexin V-FITC and PI for $15 \mathrm{~min}$. The cells were analyzed with Beckman CXP software on a FC-500 flow cytometer (Beckman Coulter, Pasadena, CA, USA) within $1 \mathrm{~h}$ of cell collection.

\section{DNA constructs and luciferase reporter assay}

The 3'-UTR sequence of DRAM2 predicted to interact with $\mathrm{miR}-125 \mathrm{~b}$ or a mutated sequence within the predicted target sites, and inserted into the $\mathrm{XbaI} / \mathrm{FseI}$ sites of the pGL3 control vector (Promega, Madison, WI, USA). The mutant 3'UTR of DRAM2 (various nucleotides within the binding sites were mutated) was amplified using DRAM2 3'UTR as the template. For the luciferase activity analysis, cells were co-transfected with the miR-125b mimic and DRAM2 3'UTR, or mutant 3'UTR, together with the controls. The DharmaFECT Duo transfection reagent (Thermo Fisher Scientific, Waltham, MA, USA) was used for $48 \mathrm{~h}$, and the luciferase assays were performed with the Dual-Glo Luciferase assay (Promega, Beijing, China) according to the manufacturer's instructions.

\section{Western blot assay}

Total protein was first separated by $12 \%$ SDSpolyacrylamide gel electrophoresis and then transferred 
to nitrocellulose membranes (Millipore, Boston, MA, USA). The membranes were blocked with $5 \%$ nonfat milk and incubated with a rabbit anti-DRAM2 polyclonal antibody at a dilution of 1:200 (SigmaAldrich), or a mouse anti- $\beta$-actin monoclonal antibody at a dilution of 1:2000 (Sigma, San Francisco, CA, USA). The membranes were subsequently incubated with a goat anti-mouse or anti-rabbit horseradish peroxidase secondary antibody (Sigma). The protein complexes were detected using the enhanced chemiluminescence reagents (Pierce, Rockford, IL, USA) and $\beta$-actin was used as an internal control.

\section{Statistical analysis}

Continuous variables are expressed as the mean \pm SD. Measurement data were analyzed using Student's $t$-test, whereas categorical data were examined using the $\chi^{2}$-test. $P$-values $<0.05$ were considered to indicate statistically significant differences. All statistical analyses were performed using SPSS 16.0 software (SPSS; Chicago, IL, USA).

\section{Results}

\section{MiR-125b is upregulated in retinoblastoma tissues and} cell lines

To determine the potential roles of $\mathrm{miR}-125 \mathrm{~b}$ in retinoblastoma, we used RT-PCR to examine the expression level of miR-125b in an expanded cohort of 38 retinoblastoma patients. The statistical results were consistent with the expression microarray data, and miR-125b expression was remarkably upregulated in retinoblastoma tissues compared with the corresponding adjacent NCTs (Figure 1a). To further identify that this phenomenon is consistent and common in retinoblastoma cell lines, the expression of miR-125b was detected by RTPCR in a panel of three retinoblastoma cell lines and a normal human retinal pigment epithelial cell line (ARPE-19). As shown in Figure 1b, the expression of miR-125b was significantly higher in cancer cells than that in the normal control. These data show that the expression of miR-125b is universally upregulated in retinoblastoma cells and patients, indicating that decreased $\mathrm{miR}-125 \mathrm{~b}$ expression might contribute as a tumor suppressor and prevent tumor development.

\section{Overexpression miR-125b directly promotes cell proliferation}

To further investigate the role of miR-125b in retinoblastoma cell proliferation, $\mathrm{miR}-125 \mathrm{~b}$ was overexpressed or repressed in HXO-Rb44 cells and Y79 cells by transfection with miR-125b mimic or miR-125b inhibitor (Figures 2a and b). The MTT assay showed that overexpression of miR-125b accelerated cell

proliferation. Conversely, downregulated expression of miR-125b delayed cell proliferation in comparison with the control group (Figures $2 \mathrm{c}$ and d). Similar data were also obtained in one other cell line (data not shown). These results indicate that miR-125b has an important role in regulating the proliferation of retinoblastoma cells.

\section{Ectopic expression of miR-125b directly affects cell apoptosis, migration, and invasion in vitro}

One of the hallmark of cancer is its ability to evade apoptosis. $^{28}$ Thus, we next investigated the effect of miR-125b on retinoblastoma cell apoptosis using the
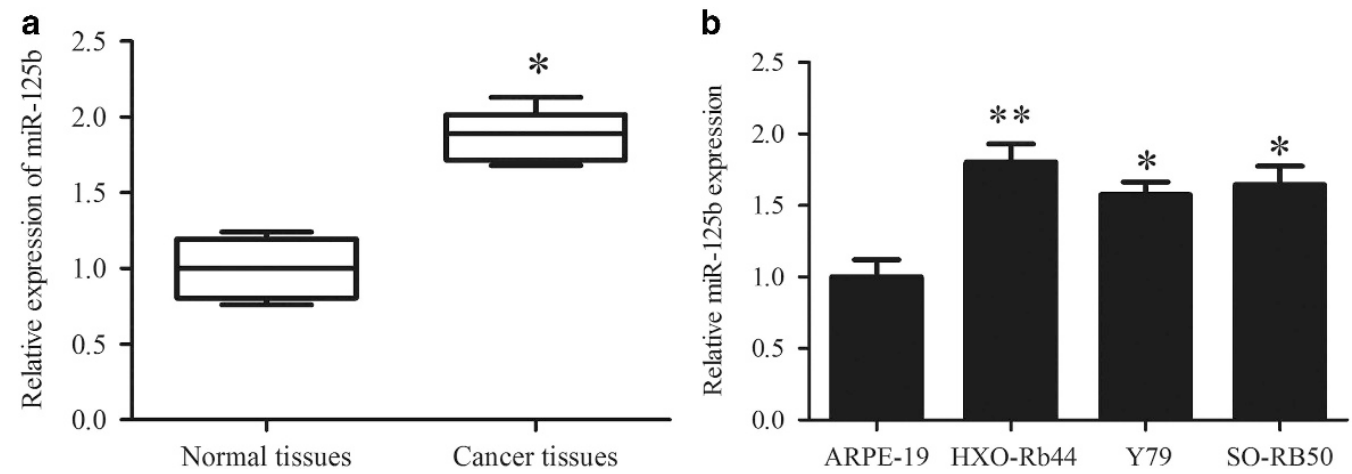

Figure 1 MiR-125b expression is frequently upregulated in retinoblastoma tissues and cell lines. (a) The expression level of mature miR-125b in retinoblastoma tissues and their matched adjacent noncancerous tissues were determined by RT-PCR. (b) The expression level of miR-125b in three retinoblastoma cell lines and normal human retinal pigment epithelial cell line ARPE-19 were checked by RTPCR. Data are representative of three experiments. Error bars represent mean $\pm S D$, and the symbols ** and * indicate differences from their respective controls at $P<0.01$ and $P<0.05$, respectively. 

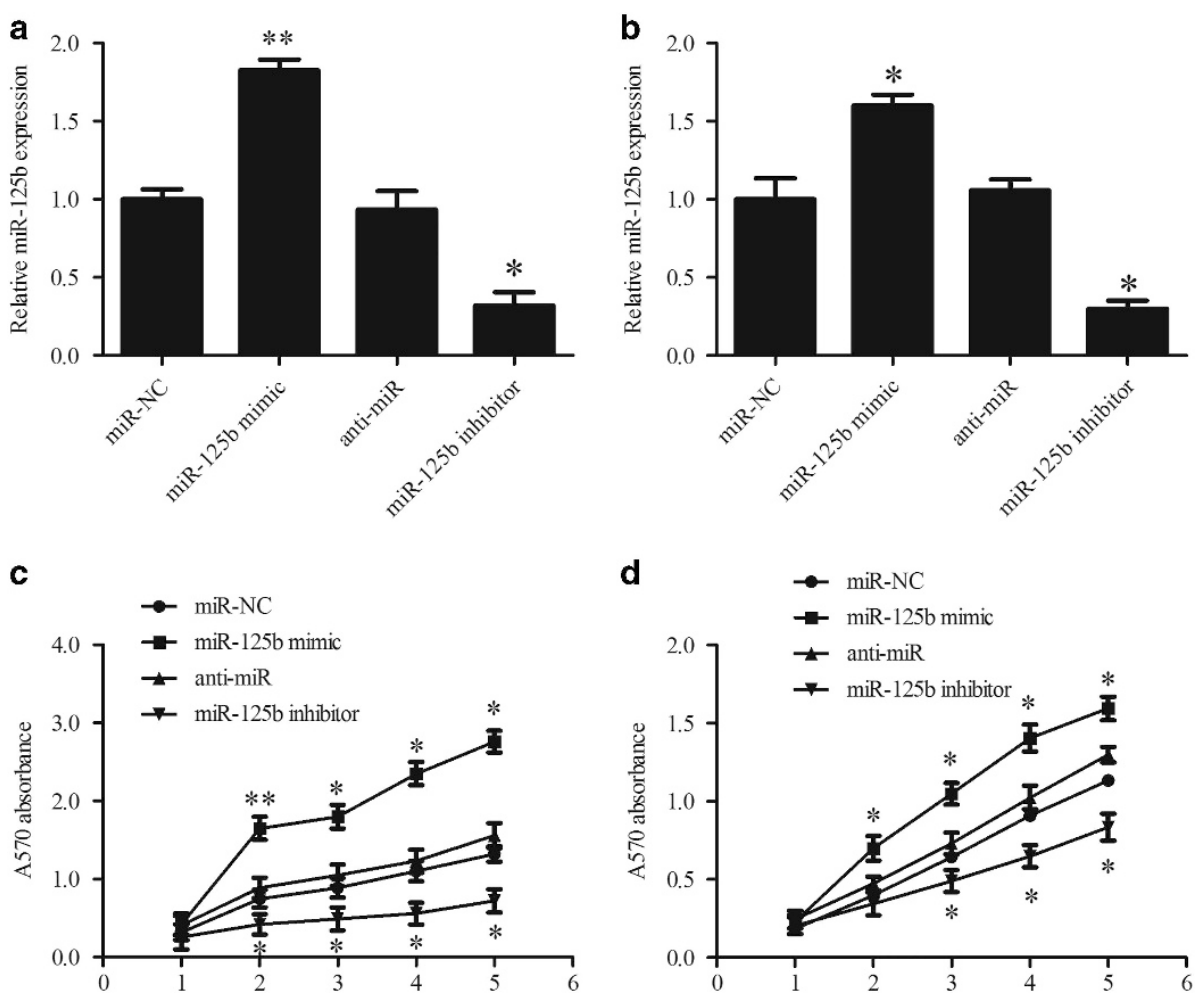

Figure 2 MiR-125b affects retinoblastoma cell proliferation. (a) Expression of miR-125b in HXO-Rb44 cells transfected with the miR-125b mimic or miR-125b inhibitor compared with their negative controls were quantified by RT-PCR. (b) Expression of miR-125b in Y79 cells was quantified by RT-PCR. (c) Proliferation rates of HXO-Rb44 cells were detected by the MTT assay. (d) Proliferation rates of Y79 cells were detected by the MTT assay. The symbols ** and * indicate differences from their respective controls at $P<0.01$ and $P<0.05$, respectively.

Annexin V-FITC kit. As shown in Figure 3a, overexpression of miR-125b significantly decreased apoptosis in HXO-Rb44 cells. The percentage of apoptotic cells decreased to 6.8 after transient transfection with the miR-125b mimic. Suppressed expression of miR-125b increased apoptosis in HXO-Rb44 cells (Figure 3b). Overall, these data indicate that miR-125b may have a function of inhibiting apoptosis in retinoblastoma cells.

To analyze the role of miR-125b in cell migration and invasion, which are the key determinants of malignant progression and metastasis, wound-healing and Transwell assays were performed in HXO-Rb44 cells. Cells treated with the miR-125b mimic were distinctively more migratory, and cells treated with the miR-125b inhibitor were distinctively less migratory than scramble control treated or untreated cells at $48 \mathrm{~h}$ after scratching (Figure 3c). Furthermore, we conducted cell invasion assays to measure the directional invasion ability following the ectopic expression of miR-125b in HXO-Rb44 cells. As shown in Figure 3d, the invasiveness of cells transfected with the miR-125b mimic was markedly increased and, conversely, the invasiveness of cells transfected with the miR-125b inhibitor was markedly decreased compared with the scramble control and untreated cells.

\section{DRAM2 is direct target genes of miR-125b}

The results from the luciferase reporter assay demonstrate that miR-125b increased the luciferase intensity of wildtype DRAM2 3'UTR by $\sim 85 \%$ compared with the normal cells. However, there was no change in luciferase activity in DRAM2 3'UTR-mut groups, indicating that DRAM2 is a direct target of miR-125b (Figure 4a). To identify the targeting of DRAM2 by miR-125b, RT-PCR was used to examine the DRAM2 mRNA level after the overexpression of miR-125b. As predicted, the overexpression of miR-125b in HXO-Rb44 cells decreased the level of DRAM2 mRNA (Figure $4 \mathrm{~b}$ ). Western blotting was used to examine the DRAM2 protein level after the overexpression of miR-125b, which showed that overexpression of miR-125b in HXORb44 cells decreased the level of DRAM2 protein (Figure 4c). These data indicate that DRAM2 might be a potential miR-125b target gene.

MiR-125b promotes cell proliferation, migration, invasion, and represses cell apoptosis by targeting DRAM2 in retinoblastoma

To further evaluate whether DRAM2 is a functional target of miR-125b in retinoblastoma cells, 
a

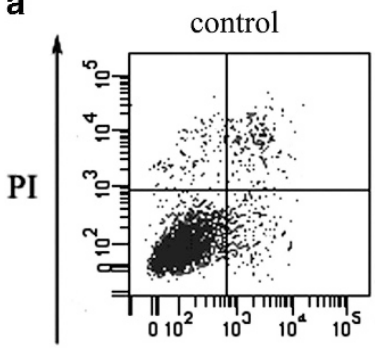

miR-125b mimic

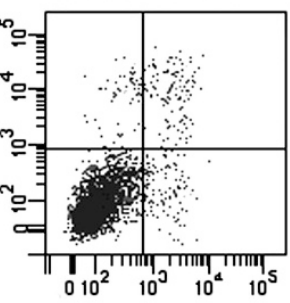

miR-125b inhibitor

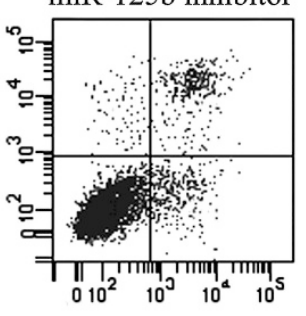

b

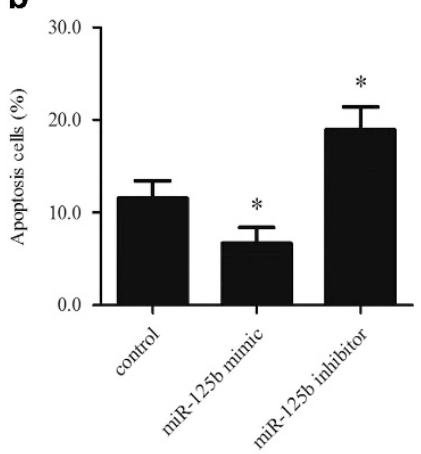

C

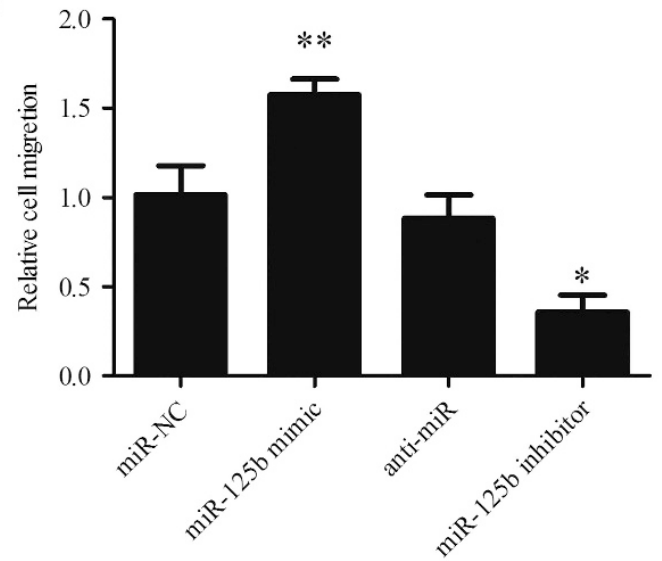

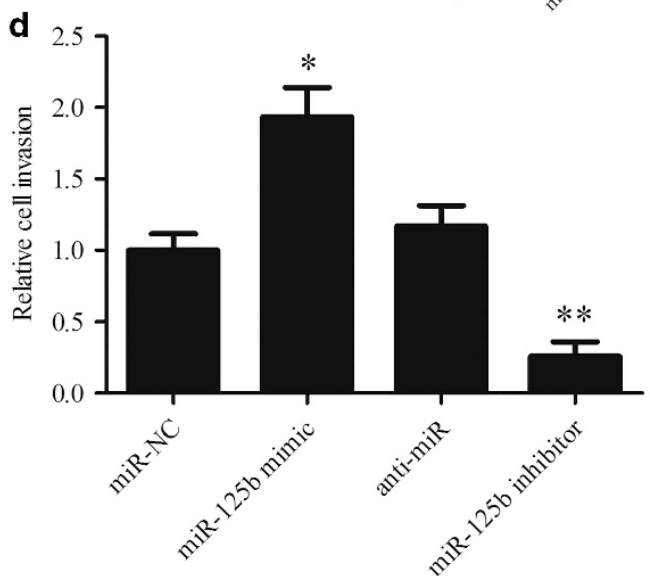

Figure 3 MiR-125b affects retinoblastoma cell migration, invasion, and apoptosis in vitro. (a) The effect of miR-125b apoptosis was examined by flow cytometric analysis. Cells were analyzed for apoptotic rate after staining with Annexin V-FITC and PI staining, as described in the Materials and Methods section. (b) Quantification of relative apoptotic in HXO-Rb44 cells transfected with miR-125b mimic or miR-125b inhibitor for $48 \mathrm{~h}$. Data represent mean \pm SD from three independent experiments. (c) Wound-healing assays were used to analyze the effect of miR-125b on cell migration of HXO-Rb44 cells. (d) Transwell assays were used to analyze the effect of miR-125b on cell invasion of HXO-Rb44 cells. The symbols ** and * indicate differences from their respective controls at $P<0.01$ and $P<0.05$, respectively.

pcDNA-3.1-DRAM2 was transfected into HXO-Rb44 cells with the miR-125b mimic to construct a DRAM2 overexpression model. After a series of functional restoration assays, we found that overexpressed DRAM2 reversed the promotion of cell proliferation, migration, and invasion induced by miR-125b (Figures 5a-c). Moreover, DRAM2 overexpression significantly increased the apoptosis rate (Figure $5 \mathrm{~d}$ ). Collectively, these data show that miR-125b promotes retinoblastoma cell proliferation and invasion, and represses apoptosis by directly targeting DRAM2.

\section{Discussion}

In this study, we analyzed the expression of miR-125b in 38 retinoblastoma patients, and three retinoblastoma cell lines, HXO-Rb44, Y79, and SO-RB50. We found that the levels of this miRNA were much higher in retinoblastoma tissues and cell lines compared with adjacent controls.
These results allowed us to speculate that the silencing of miR-125b may confer a survival advantage to retinoblastoma patients. Overexpression of miR-125b by transfection with an miRNA mimic promoted cell proliferation. As metastasis is the major cause of morbidity and mortality in retinoblastoma patients, we investigated the roles of miR-125b in cell migration and invasion of retinoblastoma cells. Furthermore, we identified DRAM2 was a direct target of miR-125b and showed that miR-125b functions as a tumor oncogene gene by downregulating DRAM2 expression, providing a potential diagnostic and therapeutic target for the treatment of retinoblastoma.

DRAM2 is the most homologous protein to DRAM among the DRAM-like proteins. ${ }^{24}$ DRAM (DNA-damageregulated autophagy modulator) is a crucial component of p53-mediated cell death, and the induction of autophagy by DRAM is a potential mechanism contributing to cell death. ${ }^{23}$ Both DRAM2 and DRAM are predicted to have six transmembrane domains, and 


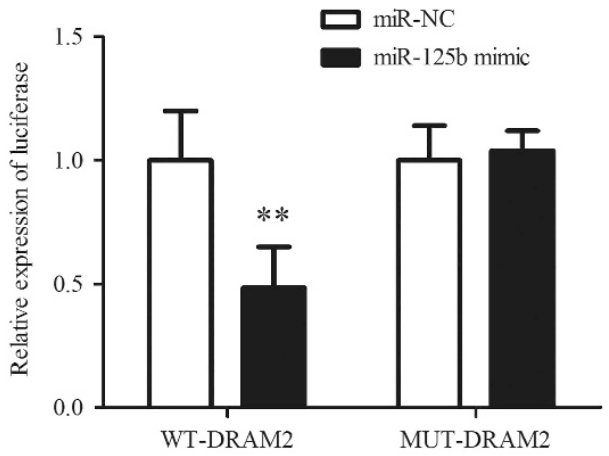

\section{C}
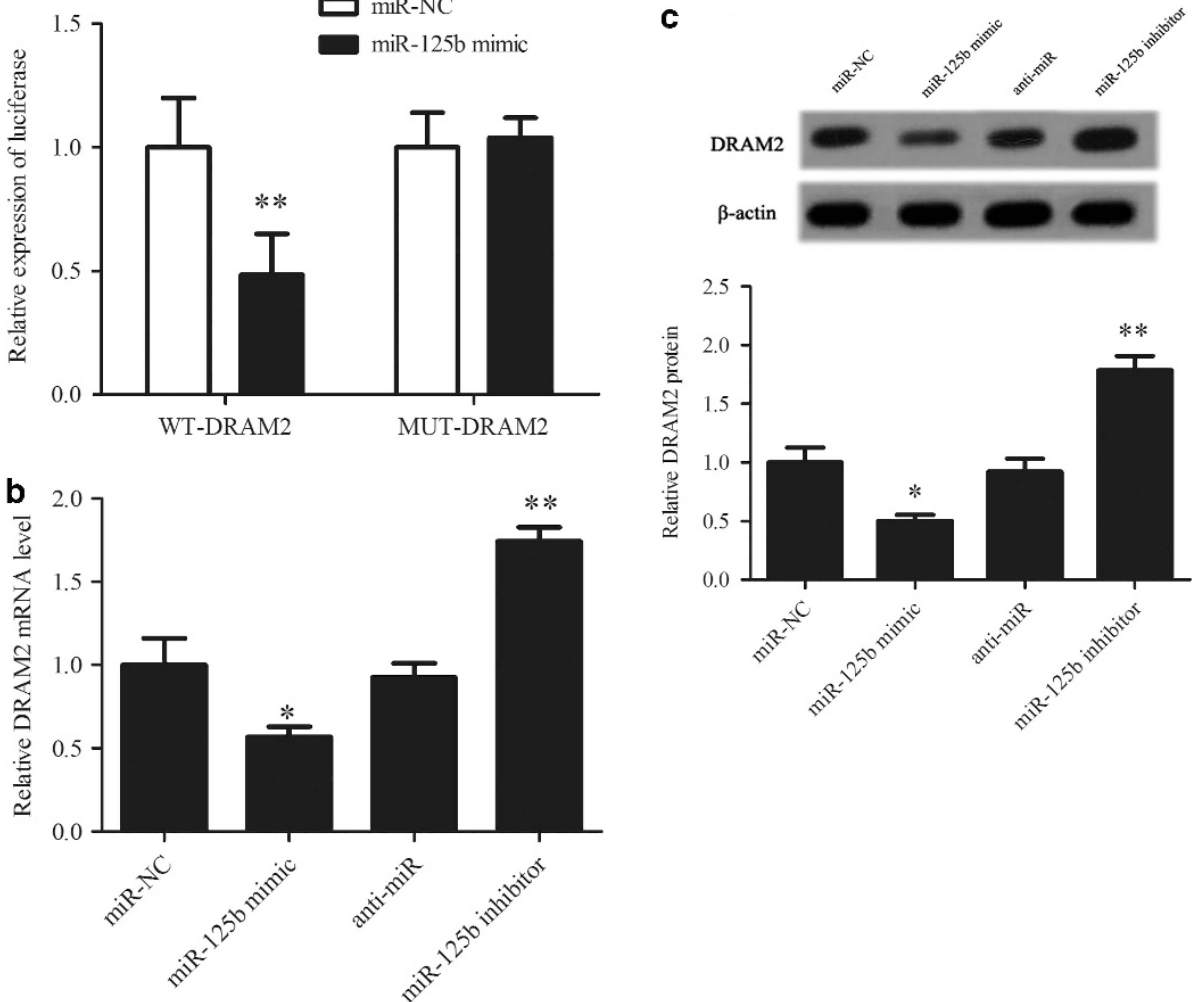

Figure 4 MiR-125b can lead to downregulated DRAM2 expression by directly targeting the DRAM2 3'UTR. (a) Relative luciferase activity of $\mathrm{HXO}-\mathrm{Rb} 44$ cells co-transfected with increasing amounts of miR-125b mimic and DRAM2 3'UTR-luciferase reporter vector that contained a wild-type sequence (DRAM2-3'UTR-wt), or in a vector that contained a mutant sequence (DRAM2-3'UTR-mut) within the miR-125b-binding site. (b) RT-PCR analysis of DRAM2 expression in miR-125b mimic-/inhibitor-transfected cells or their negative controls $48 \mathrm{~h}$ after cells were transfected. (c) Western blotting analysis of DRAM2 expression in miR-125b mimic-/inhibitor-transfected cells or their negative controls. Bars represent the mean \pm SD of three independent experiments. The symbols ${ }^{* *}$ and ${ }^{*}$ indicate differences from their respective controls at $P<0.01$ and $P<0.05$, respectively.

DRAM2 shares $36.7 \%$ amino acid similarity with DRAM. ${ }^{24}$ It has been reported that DRAM2 expression is frequently downregulated in retinoblastoma and that overexpression of DRAM2 induces apoptosis. ${ }^{29,30}$ As many proapoptotic proteins are downregulated in tumor cells, the expression of DRAM2 RNA was generally downregulated in the retinoblastoma samples that we examined, compared with the matched normal tissues.

In this study, we generated an anti-DRAM2 antibody; western blot analysis with this antibody confirmed that DRAM2 protein is downregulated in retinoblastoma (Figure 4c). We report here that DRAM2 is a direct target gene of miR-125b, and thus is markedly downregulated by miR-125b at the posttranscriptional level in retinoblastoma. Downregulation of miR-125b or promoting gene expression resulted in a significant reduction in cancer cell proliferation and migration in vitro. Our work broadens our understanding of the roles that miR-125b has in retinoblastoma by targeting several different genes, and thus indicates that it can be a powerful target for cancer therapy.

In conclusion, the current study provides new evidence that miR-125b may function as a tumor promoter for retinoblastoma by regulating the expression of DRAM2. Furthermore, higher expression of miR-125b in retinoblastoma specimens was correlated with cell migration and invasion. Our findings regarding miR-125b are encouraging and suggest that this miRNA could be a potential target for the treatment of retinoblastoma in the future. Our work broadens our understanding of the roles that $\mathrm{miR}-125 \mathrm{~b}$ has in retinoblastoma by targeting several different genes, and thus may be a powerful target for cancer therapy. 
a

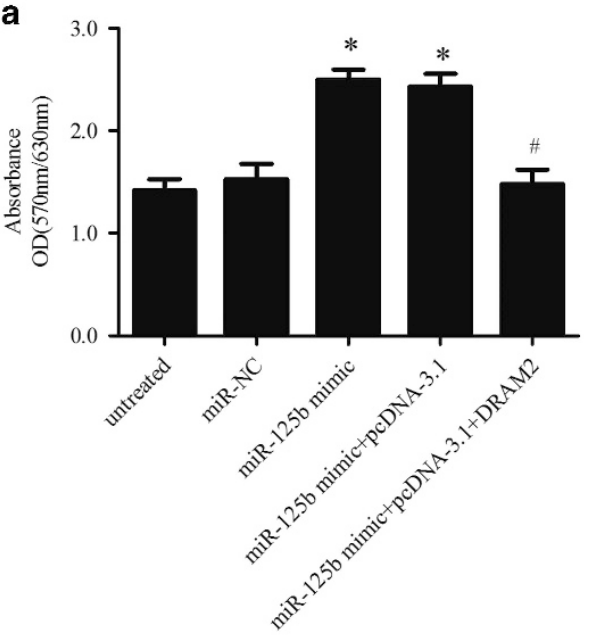

C

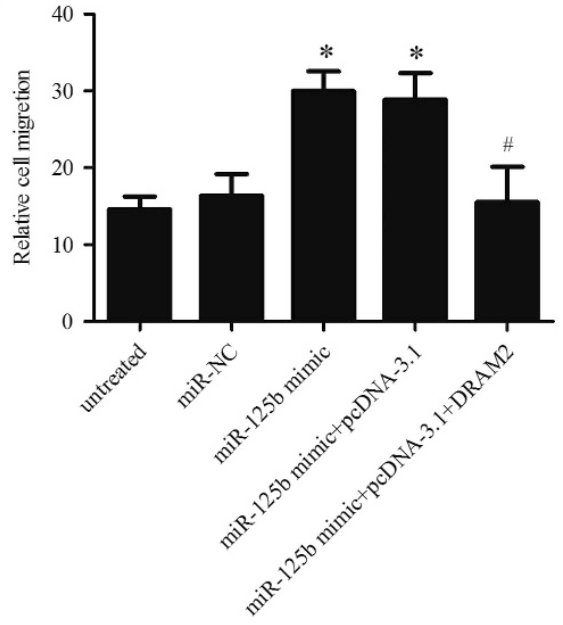

b

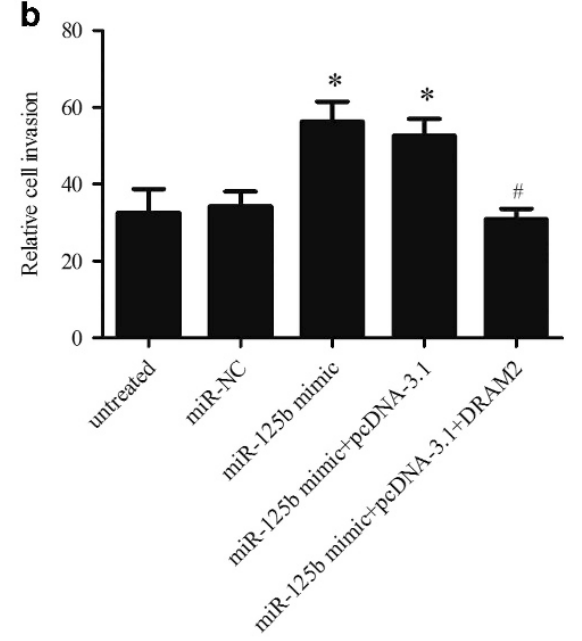

d

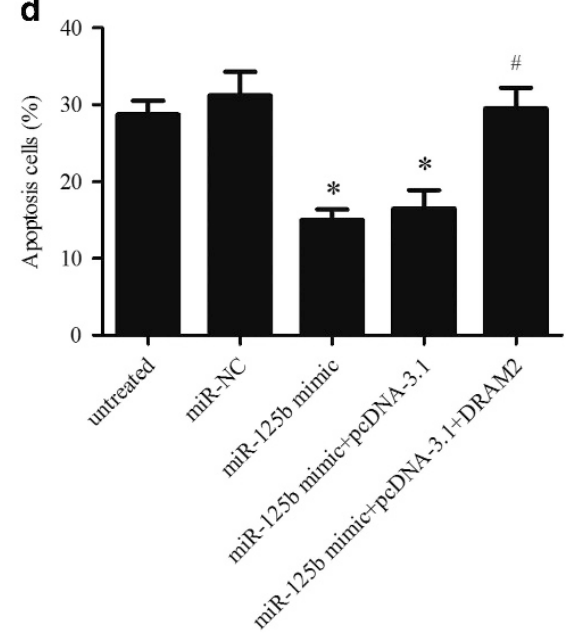

Figure 5 MiR-125b regulates retinoblastoma cell development by targeting DRAM2 expression. (a) The MTT assay showed that overexpression of DRAM2 rescues the effect of miR-125b in the regulation of proliferation. (b) Upregulation of DRAM2 inhibited cell migration, and decreased expression of miR-125b led to the opposite result. (c) Upregulation of the DRAM2 inhibited cell invasion and abrogated miR-125b-promoted invasion in retinoblastoma cells. (d) Upregulation of DRAM2 promoted cell apoptosis induced by miR-125b in retinoblastoma cells. Data presented are mean $\pm \mathrm{SD},{ }^{*}$ indicates $P<0.05$ compared with normal control, and ${ }^{*}$ indicates $P<0.05$ compared with miR-125b mimic+pcDNA-3.1.

\section{Summary}

\section{What was known before}

- It is known that microRNAs (miRNAs) are a class of small, noncoding RNAs that act as key regulators in various physiological and pathological processes. However, the regulatory mechanisms involving miRNAs in retinoblastoma remain largely unknown.

What this study adds

- Our data collectively demonstrate that miR-125b is a suppressor gene miRNA that can promote retinoblastoma cell proliferation and migration by downregulating the suppressor gene DRAM2, indicating that miR-125b may represent a new potential diagnostic and therapeutic target for retinoblastoma treatment.

\section{Conflict of interest}

The authors declare no conflict of interest.

\section{Acknowledgements}

We are grateful to all the patients and individuals in the study who made this work possible. We thank the clinicians and hospital staff who contributed to data collection for this study. This study was funded by the National Natural Science Foundation (No. 81302198). All procedures performed in studies involving human participants were in accordance with the ethical standards of the institutional and/or national research committee, 
and with the 1964 Helsinki declaration and its later amendments, or comparable ethical standards. Informed consent was obtained from all individual participants included in the study.

\section{References}

1 Dimaras H, Kimani K, Dimba EA, Gronsdahl P, White A, Chan HS et al. Retinoblastoma. Lancet 2012; 379(9824): 1436-1446.

2 Kivela T. The epidemiological challenge of the most frequent eye cancer: retinoblastoma, an issue of birth and death. Br J Ophthalmol 2009; 93(9): 1129-1131.

3 Houston SK, Murray TG, Wolfe SQ, Fernandes CE. Current update on retinoblastoma. Int Ophthalmol Clin 2011; 51(1): 77.

4 Xu X, Ge S, Jia R, Zhou Y, Song X, Zhang H et al. Hypoxia-induced miR-181b enhances angiogenesis of retinoblastoma cells by targeting PDCD10 and GATA6. Oncol Rep 2015; 33(6): 2789-2796.

5 Wang T, Liu Y, Yuan W, Zhang L, Zhang Y, Wang Z et al. Identification of microRNAome in rat bladder reveals miR-1949 as a potential inducer of bladder cancer following spinal cord injury. Mol Med Rep 2015; 12(2): 2849-2857.

6 Chen K, Rajewsky N. The evolution of gene regulation by transcription factors and microRNAs. Nat Rev Genet 2007; 8(2): 93-103.

7 Du T. Beginning to understand microRNA function. Cell Res 2007; 17(8): 661-663.

8 Bartel DP. MicroRNAs: target recognition and regulatory functions. Cell 2009; 136(2): 215-233.

9 Chang-Zheng C, Ling L, Lodish HF, Bartel DP. MicroRNAs modulate hematopoietic lineage differentiation. Science 2004; 303(5654): 83-86.

10 Brennecke J, Hipfner DR, Stark A, Russell RB, Cohen SM. bantam encodes a developmentally regulated microRNA that controls cell proliferation and regulates the proapoptotic gene hid in Drosophila. Cell 2003; 113: 25-36.

11 Xu P, Vernooy SY, Guo M, Hay BA. The Drosophila microRNA Mir-14 suppresses cell death and is required for normal fat metabolism. Curr Biol 2003; 13(9): 790-795.

12 Victor A. The functions of animal microRNAs. Nature 2004; 431(7006): 350-355.

13 Shenouda SK, Alahari SK. MicroRNA function in cancer: oncogene or a tumor suppressor? Cancer Metastasis Rev 2009; 28(3-4): 369-378.

14 Shipeng F, Shujie C, Xin Z, Xichen B, Wei W, Huiping L et al. MicroRNA-192 targeting retinoblastoma 1 inhibits cell proliferation and induces cell apoptosis in lung cancer cells. Nucleic Acids Res 2011; 39(15): 6669-6678.

15 Dalgard CL, Gonzalez M, O'Brien JM. Differential microRNA-34a expression and tumor suppressor function in retinoblastoma cells. Invest Ophthalmol Vis Sci 2009; 50(10): 4542-4551.

16 Jo DH, Kim JH, Park W-Y, Kim K-W, Yu YS, Kim JH. Differential profiles of microRNAs in retinoblastoma cell lines of different proliferation and adherence patterns. J Pediatr Hematol Oncol 2011; 33(7): 529-533.

17 Zhang Y, Wu JH, Han F, Huang JM, Shi SY, Gu RD et al. Arsenic trioxide induced apoptosis in retinoblastoma cells by abnormal expression of microRNA-376a. Neoplasma 2013; 60(3):247-253.

18 Xu BS, Ling RX, Ai HM, Clifford GT, Hsing JK, Ralph WW. miR-125b promotes growth of prostate cancer xenograft tumor through targeting pro-apoptotic genes. Prostate 2011; 71(5): 538-549.

19 Li Q, Han Y, Wang C, Shan S, Wang Y, Zhang J et al. MicroRNA-125b promotes tumor metastasis through targeting tumor protein 53-induced nuclear protein 1 in patients with non-small-cell lung cancer. Cancer Cell Int 2015; 15(1): 1-10.

$20 \mathrm{Gu}$ WL, Ye DX, Wu JJ. [Expression and clinical significance of plasma microRNA-125b level in patients with oral squamous cell carcinoma]. Shanghai Kou Qiang Yi Xue 2015; 24: 71-75.

21 Zhao J-J, Yang J, Lin J, Yao N, Zhu Y, Zheng J et al. Identification of miRNAs associated with tumorigenesis of retinoblastoma by miRNA microarray analysis. Childs Nerv Syst 2009; 25(1): 13-20.

22 To K-H, Pajovic S, Gallie BL, Thériault BL. Regulation of p14ARF expression by miR-24: a potential mechanism compromising the p53 response during retinoblastoma development. BMC Cancer 2012; 12(1): 1.

23 El-Asrag ME, Sergouniotis PI, McKibbin M, Plagnol V, Sheridan E, Waseem $\mathrm{N}$ et al. Biallelic mutations in the autophagy regulator DRAM2 cause retinal dystrophy with early macular involvement. Am J Hum Genet 2015; 96(6): 948-954.

24 Park S-M, Kim K, Lee E-J, Kim B-K, Lee TJ, Seo T et al. Reduced expression of DRAM2/TMEM77 in tumor cells interferes with cell death. Biochem Biophys Res Commun 2009; 390(4): 1340-1344.

25 Peracchio C, Alabiso O, Valente G, Isidoro C. Involvement of autophagy in ovarian cancer: a working hypothesis. J Ovarian Res 2012; 5(1): 1.

26 Tolosa L, Donato MT, Gómez-Lechón MJ. General cytotoxicity assessment by means of the MTT assay. Methods Mol Biol 2015; 1250: 333-348.

27 Lin Z, Liu X, Jin H, Guo X, Xia L, Chen Z et al. miR-206 inhibits gastric cancer proliferation in part by repressing cyclinD2. Cancer Lett 2013; 332(1): 94-101.

28 Cotter TG. Apoptosis and cancer: the genesis of a research field. Nat Rev Cancer 2009; 9(7): 501-507.

29 Ryan KM. p53 and autophagy in cancer: guardian of the genome meets guardian of the proteome. Eur J Cancer 2011; 47(1): 44-50.

30 Marlhens F, Griffoin JM, Bareil C, Arnaud B, Claustres M, Hamel CP. Autosomal recessive retinal dystrophy associated with two novel mutations in the RPE65 gene. Eur J Hum Genet 1998; 6(5): 527-531. 